Proceedings of the 16th Czech and Slovak Conference on Magnetism, Košice, Slovakia, June 13-17, 2016

\title{
The Energy Spectrum and Thermodynamics of Spin-1/2 XX Chain with Ising Impurities
}

\author{
E.V. EZERSKAYA* \\ Faculty of Physics, V.N. Karazin Kharkiv National University, Svoboda Sq. 4, 61022 Kharkiv, Ukraine \\ The energy spectrum and peculiarities of field and temperature dependences of the basic thermodynamic \\ characteristics of the finite spin- $1 / 2 \mathrm{XX}$ chain closed by one $z z$ (Ising) bond and open ends XX-chain with two \\ $z z$-impurities at the both ends have been investigated.
}

DOI: 10.12693/APhysPolA.131.928

PACS/topics: 75.10.Jm, 75.40.Cx

\section{Introduction}

Low-dimensional spin models occupy special place in quantum theory of magnetism due to the fact that such systems in some cases may have exact analytical solutions $[1,2]$. Usually, the real magnets are characterized by different types of structure defects, so the theoretical study of the influence of impurities on the energy spectrum and the thermodynamics of spin chains with heterogeneity is of great interest.

\section{The models}

We study the energy spectrum and the thermodynamics of two exactly solvable spin models: finite spin- $1 / 2$ XX-chain with periodic boundaries ("ring") closed by the Ising spin $S_{1}$ and open ends finite XX chain ("line") with two different edge impurity Ising spins $S_{1}, S_{2}$. Model Hamiltonians have the form

$$
\begin{aligned}
& \widehat{H}_{\text {ring }}=-h_{0} S_{0}^{z}-J_{0}\left(S_{1}^{z}+S_{N}^{z}\right) S_{0}^{z}-h \sum_{n=1}^{N} S_{n}^{z} \\
& -J \sum_{n=1}^{N-1}\left(S_{n}^{x} S_{n+1}^{x}+S_{n}^{y} S_{n+1}^{y}\right), \\
& \widehat{H}_{\text {line }}=-\left(h_{0} S_{0}^{z}+h_{N+1} S_{N+1}^{z}\right)-J_{0} S_{0}^{z} S_{1}^{z} \\
& -J_{N+1} S_{N}^{z} S_{N+1}^{z}-h \sum_{n=1}^{N} S_{n}^{z} \\
& -J \sum_{n=1}^{N-1}\left(S_{n}^{x} S_{n+1}^{x}+S_{n}^{y} S_{n+1}^{y}\right)
\end{aligned}
$$

for "ring" and for "line", respectively. Here $J>0$ is the exchange integral for spin- $1 / 2 \mathrm{XX}$ chain, $J_{0}, J_{N+1}$ are the exchange integrals of the Ising (or $z z$ )-type interactions with impurity spins, $h=g \mu_{\mathrm{B}} H, h_{0}=g_{0} \mu_{\mathrm{B}} H, h_{N+1}=$ $g_{N+1} \mu_{\mathrm{B}} H, \mu_{\mathrm{B}}$ is the Bohr magneton, $g, g_{0}$ and $g_{N+1}$ are $g$-factors for chain and impurities respectively, $H$ is

\footnotetext{
*e-mail: yezerska@karazin.ua
}

the longitudinal uniform magnetic field. Positive and negative values of the Ising interactions correspond to ferromagnetic (FM) and antiferromagnetic (AF) type of exchange. $Z$-projections of the impurity spins $(\sigma=$ $\left.-S_{i}, \ldots, S_{i}, i=1,2\right)$ are the good quantum numbers, so Hamiltonians (1) and (2) may be treated as a set of independent Hamiltonians $\widehat{H}\left(\sigma_{0}\right), \widehat{H}\left(\sigma_{0}, \sigma_{N+1}\right)$ of finite spin-1/2 XX chains with the effective "impurity" spins $(s=1 / 2)$ at the ends. These Hamiltonians describe some kind of well-known broken-chain effect in real quasione-dimensional magnets $[3,4]$. The Ising interactions of impurity are described by additional Zeeman type terms. Spin-1/2 XX chain is a well-known example of an exactly solvable spin system $[1,2]$. The Jordan-Wigner transformation [1] reduces each of $\widehat{H}\left(\sigma_{0}\right), \widehat{H}\left(\sigma_{0}, \sigma_{N+1}\right)$ to the ideal gas of spinless fermions

$$
\begin{aligned}
& \widehat{H}_{\text {ring }}\left(\sigma_{0}\right)=E_{0}\left(\sigma_{0}\right) \\
& +\left(h+J_{0} \sigma_{0}\right)\left(a_{1}^{\dagger} a_{1}+a_{N}^{\dagger} a_{N}\right)+h \sum_{n=2}^{N-1} a_{n}^{\dagger} a_{n} \\
& \quad-\frac{J}{2} \sum_{n=1}^{N-1}\left(a_{n}^{\dagger} a_{n+1}+a_{n+1}^{\dagger} a_{n}\right) ; \\
& E_{\text {ferro }}\left(\sigma_{0}\right)=-\left(h_{0}+J_{0}\right) \sigma_{0}-\frac{h N}{2}, \\
& \quad \sigma_{0}=-S_{1}, \ldots, S_{1}, \\
& \widehat{H}_{\text {line }}\left(\sigma_{0}, \sigma_{N+1}\right)=E_{f e r r o}\left(\sigma_{0}, \sigma_{N+1}\right) \\
& \quad+\left(h+J_{0} \sigma_{0}\right) a_{1}^{\dagger} a_{1}+\left(h+J_{N+1} \sigma_{N+1}\right) a_{N}^{\dagger} a_{N} \\
& \quad+h \sum_{n=2}^{N-1} a_{n}^{\dagger} a_{n}-\frac{J}{2} \sum_{n=1}^{N-1}\left(a_{n}^{\dagger} a_{n+1}+a_{n+1}^{\dagger} a_{n}\right) ; \\
& E_{\text {ferro }}\left(\sigma_{0}, \sigma_{N+1}\right)=-\left(h_{0}+\frac{J_{0}}{2}\right) \sigma_{0} \\
& \quad-\left(h_{N+1}+\frac{J_{N+1}}{2}\right) \sigma_{N+1}-\frac{h N}{2}, \\
& \sigma_{0}=-S_{1}, \ldots, S_{1}, \sigma_{N+1}=-S_{2}, \ldots, S_{2} .
\end{aligned}
$$

We diagonalized Hamiltonians (3) and (4) by solving oneparticle Schrödinger equation in the lattice site representation and derived the following dispersion relations for 
the exact energy spectrum for the "ring":

$$
\frac{1 \pm x_{\sigma}^{N+1}}{x_{\sigma}\left(1 \pm x_{\sigma}^{N-1}\right)}=\alpha_{0}
$$

and for the "line"

$$
\begin{aligned}
& \left(\alpha_{0}+\frac{1}{x_{\sigma}}\right)\left(\alpha_{N+1}+\frac{1}{x_{\sigma}}\right) \\
& -\left(\alpha_{0}+x_{\sigma}\right)\left(\alpha_{N+1}+x_{\sigma}\right) x_{\sigma}^{2(N-1)}=0,
\end{aligned}
$$

with the "excitation" energies

$$
\varepsilon\left(x_{\sigma}\right)=h-\frac{J}{2}\left(x_{\sigma}+\frac{1}{x_{\sigma}}\right)
$$

and

$$
\begin{aligned}
\alpha_{0} & =\frac{2 J_{0} \sigma_{0}}{J}, \alpha_{N+1}=\frac{2 J_{N+1} \sigma_{N+1}}{J}, \\
\sigma_{0} & =-S_{1}, \ldots,+S_{1}, \sigma_{N+1}=-S_{2}, \ldots,+S_{2} .
\end{aligned}
$$

Quasi-continuous spectrum for $x_{\sigma}=\exp \left(\mathrm{i} k_{\sigma}\right)$ has the energy

$$
\varepsilon_{k_{\sigma}}=h-J \cos k_{\sigma}, \sigma=\sigma_{0} ; \quad \sigma=\sigma_{0}, \sigma_{N+1} .
$$

Localized impurity levels with real parameter $x(|x|<1)$ appear at sufficiently strong Ising interactions of impurities with the main chain

$$
\left|\alpha_{0}^{(1 c)}\right|>1, \quad\left|\alpha_{0}^{(2 c)}\right|>\frac{N+1}{N-1}
$$

for "ring" and the critical length for "line"

$$
\begin{aligned}
& N_{c}=\frac{\alpha_{0} \alpha_{N+1}-1}{\left(\alpha_{0} \pm 1\right)\left(\alpha_{N+1} \pm 1\right)}, \\
& \left|\alpha_{0}^{(c)}\right|>1,\left|\alpha_{N+1}^{(c)}\right|>1 \text { at } N \rightarrow \infty .
\end{aligned}
$$

\section{Thermodynamics}

The partition functions for above "ring" and "line" models are the sum of partition functions of finite XX chains with impurities described by the Hamiltonians (3) and (4), respectively

$$
\begin{aligned}
Z & =\sum_{\sigma} Z(\sigma), \quad Z(\sigma)=\exp \left(-\frac{E_{\text {ferro }}(\sigma)}{T}\right) \\
& \times \prod_{\lambda}\left[1+\exp \left(-\frac{h-J\left(x_{\lambda \sigma}^{2}+1\right) /\left(2 x_{\lambda \sigma}\right)}{T}\right)\right] .
\end{aligned}
$$

Here $\lambda$ enumerates all the solutions of dispersion relations (5) or (6), $\sigma=\sigma_{0}$ or $\sigma=\sigma_{0}, \sigma=\sigma_{0}, \sigma_{N+1}$ and $E_{\text {ferro }}(\sigma)$ from (3) or (4).

We performed the simulation of the field and the temperature dependences of the magnetization and heat capacity per spin. For AF Ising interaction the field dependence of the magnetization at very low temperatures may have a jump associated with the spin-flip of impurity spin in sufficiently strong magnetic field for "ring" and two jumps for "line". The field dependence of the specific heat for both models has a complex form with the multiple maxima. We associate additional peaks of the specific heat for AF Ising interaction of impurities with XX chain with the local impurity levels and the spin-flip of an impurity spin in strong fields. The temperature dependence of specific heat at $h=0$ for "line" is

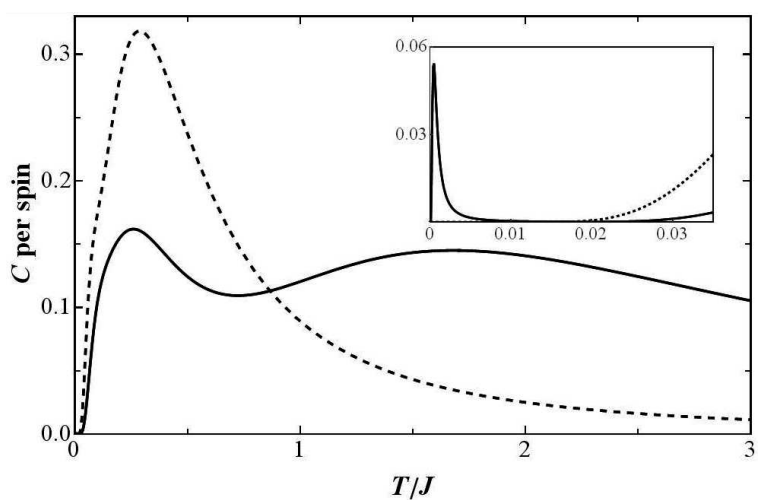

Fig. 1. Temperature dependence of specific heat $\mathrm{C}$ in dimensionless units in zero magnetic field for "line" at $N=6, J_{0} / J=-10, J_{N+1} / J=-6, S_{1}=1 / 2, S_{2}=$ 1. Dashed line indicates the specific heat for finite XX chain with the same number of spins $N=6$.

presented in Fig. 1. Additional peak at very low temperature is associated with finite size effect for short chains (see Fig. 1, inset).

One can easily calculate average values of $z$-projections of impurity spins $\left\langle S_{0}^{z}\right\rangle,\left\langle S_{N+1}^{z}\right\rangle$ and the longitudinal pair spin-spin correlation function $\left\langle S_{0}^{z} S_{N+1}^{z}\right\rangle$ for impurities in "line" from probability theory formula. To estimate the average values of $z$-projections $\left\langle S_{n}^{z}\right\rangle$ at XX chain we should find the $\left\langle S_{n}^{z}(\sigma)\right\rangle$ for a given value of $\sigma$ and then perform thermodynamic average for all possible projections of impurity spin (spins)

$$
\begin{aligned}
\left\langle S_{n}^{z}\right\rangle & =\frac{1}{2}-\frac{1}{Z} \sum_{\sigma} Z(\sigma) \sum_{\lambda}\left|A_{n \lambda}(\sigma)\right|^{2} f\left(\varepsilon_{\lambda}(\sigma)\right), \\
f(\varepsilon) & =\frac{1}{\mathrm{e}^{\varepsilon / T}+1},
\end{aligned}
$$

where $A_{n \lambda}(\sigma)$ is wave function in lattice site representation for the state with one inverted spin for Hamiltonians (3) or (4).

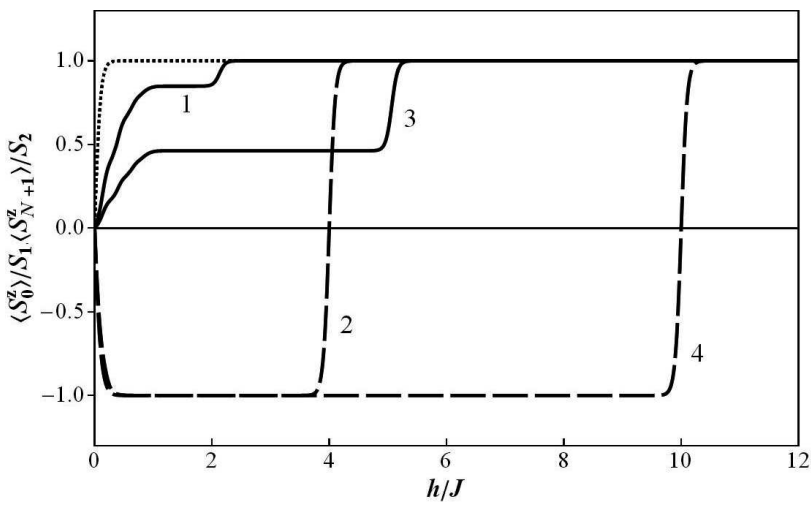

Fig. 2. Field dependence of average $z$-projections of impurity spins at $T=0.05 \mathrm{~K}$ for $N=12, S_{1}=$ $S_{2}=1 / 2, g=g_{0}=g_{N+1}=1$ : (1) "line" at $J_{0} / J=J_{N+1} / J-4$, (2) "ring" at $J_{0} / J=-4$, (3) "line" at $J_{0} / J=J_{N+1} / J=-10$, (4) "ring" at $J_{0} / J=$ -10 . Short-dashed line is the Brillouin function for free spin- $1 / 2$. 


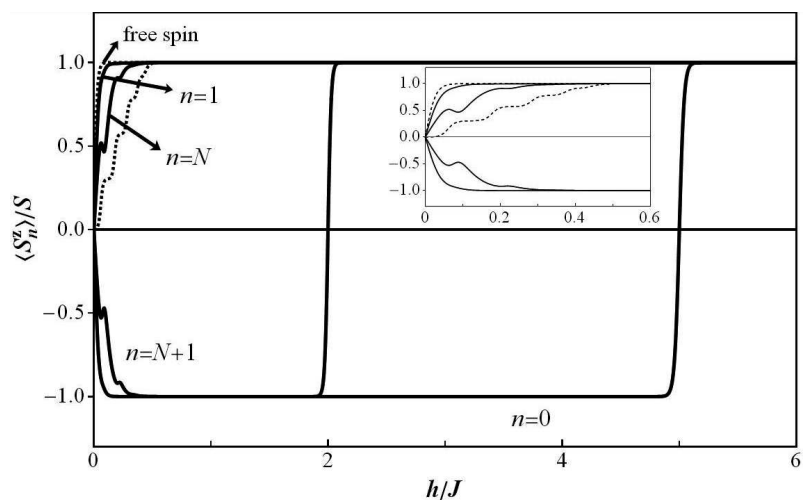

Fig. 3. Field dependence of $\left\langle S_{n}^{z}\right\rangle$ for "line" at $T=$ $0.02 \mathrm{~K}$ for $N=12, S_{1}=S_{2}=12, g=2, g_{0}=$ $1, g_{N+1}=1.5, J_{0} / J=-10, J_{N+1} / J=-6$. Shortdashed lines are for free spin-1/2 and $\left\langle S_{n}^{z}\right\rangle$ for finite XX chain with $N=12$.

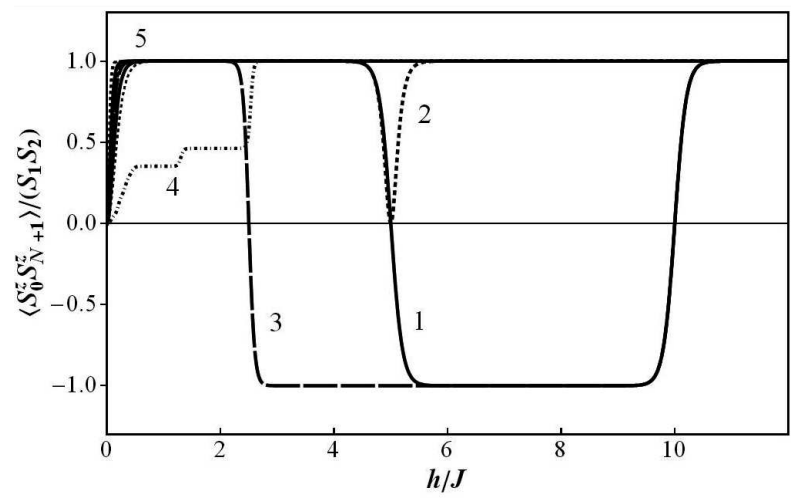

Fig. 4. Field dependence of the ratio of longitudinal pair spin-spin correlation functions to impurity spins values at $T=0.05 \mathrm{~K}$ for $N=12, S_{1}=S_{2}=$ $1 / 2, J_{0} / J=-10, J_{N+1} / J=-5, g=2$ for "line": (1) $g_{0}=g_{N+1}=0.5$, (2) $g_{0}=1, g_{N+1}=0.5$, (3) $g_{0}=0.5, g_{N+1}=1,(4) g_{0}=g_{N+1}=2$, (5) $g_{0}=g_{N+1}=2.5$. Dashed lines correspond to the product of the Brillouin functions for free spins.

The behavior of the average $z$-projection of impurity spins and longitudinal impurities spin-spin correlation functions at zero and non-zero temperature were studied numerically. It was shown that under certain conditions, the average $z$-spin projection for impurity sites at $T=0$ may have the finite jumps and non-monotonic dependence on the magnetic field at low temperatures.
The behavior of an impurity spin in closed chain and open chain can differ substantially (Fig. 2). This is due to the fact that in the closed chain the impurity spin interacts directly with the two neighboring spins. Peculiarities of this behavior are clearly visible at AF impurity interactions with the XX chain. Even for the same $g$-factors in case of the closed chain in the weak field, the impurity spin is oriented oppositely to the field. For open chain at any external fields the average $z$-projection of impurity spin is positive in this case. The behavior of $\left\langle S_{n}^{z}\right\rangle$ vs. $h / J$ for impurity spins and the spins, which are nearest to the "open chain" ends for different values of $g$-factors, is shown in Fig. 3, and here impurity spins are oriented opposite to the external field at low fields.

The longitudinal pair spin-spin correlation functions $\left\langle S_{0}^{z} S_{N+1}^{z}\right\rangle$ for the two impurity spins in "line" is shown in Fig. 4. For sufficiently strong AF Ising interactions, and $g_{0}, g_{N+1}<g$, the corresponding correlation functions may demonstrate non-monotonic field dependences at low temperatures.

\section{Conclusions}

This work is devoted to the theoretical study of quantum stationary states and thermodynamics of two onedimensional spin systems: the finite XX-chain with $z z$ (Ising) bond, closing chain in the ring and open ends XXchain with two $z z$ impurities at both ends. It is shown the possibility of an appearance of the energy levels localized near the impurity spins. The conditions for the formation of localized energy levels resulting from the strong Ising interaction between impurity spins and the main XX chain were formulated. The field and temperature dependences of the basic thermodynamic characteristics of the models were investigated.

\section{References}

[1] E. Lieb, T. Schultz, D. Mattis, Ann. Phys. 16, 407 (1961).

[2] A.A. Zvyagin, Quantum Theory of One-Dimensional Spin Systems, Cambridge Sci. Publ., Cambridge 2010.

[3] A. Klumper, D.C. Johnston, Phys. Rev. Lett. 84, 4701 (2000).

[4] Y. Liu, J.E. Drumheller, R.D. Willett, Phys. Rev. B 52, 15327 (1995). 